\title{
CONTRIBUCIÓN AL CONOCIMIENTO DEL ENDEMISMO DE LA FLORA VASCULAR EN VERACRUZ, MÉXICO
}

\author{
Gonzalo Castillo-Campos ${ }^{1}$, Ma. Elena Medina Abreo², \\ Patricia Dolores Dávila Aranda ${ }^{3}$ y José Alejandro Zavala Hurtado ${ }^{4}$ \\ ${ }^{1}$ Departamento de Biodiversidad y Sistemática, Instituto de Ecología, A.C., \\ km 2.5 carretera antigua a Coatepec No. 351, Congregación El Haya, \\ 91070 Xalapa, Veracruz, México \\ correo electrónico: castillo@ecologia.edu.mx \\ ${ }^{2}$ Departamento de Ecología Aplicada, Instituto de Ecología, A.C., \\ km 2.5 Carretera Antigua a Coatepec No. 351, Congregación El Haya, \\ 91070 Xalapa, Veracruz, México \\ correo electrónico: medinama@ecologia.edu.mx \\ ${ }^{3}$ Facultad de Estudios Superiores Iztacala, \\ Universidad Nacional Autónoma de México \\ Av. de Los Barrios s/n, 54090 Tlalnepantla, Estado de México \\ correo electrónico: pdavilaa@servidor.unam.mx \\ ${ }^{4}$ Departamento de Biología, \\ Universidad Autónoma Metropolitana-Iztapalapa, \\ Apdo. postal 55-535, 09340 México, D.F. \\ correo electrónico: jazh@xanum.uam.mx
}

\section{RESUMEN}

Se elaboró una lista de taxa endémicos de la flora vascular de Veracruz. Se registraron 94 géneros de 56 familias que contienen 131 especies y 10 taxa subespecíficos con distribución restringida a los límites del estado. Las familias con el mayor número de unidades endémicas fueron Orchidaceae (11), Myrtaceae (10), Bromeliaceae (9), Poaceae (8), Begoniaceae (7), Zamiaceae (5) y Polypodiaceae (5). Los tipos de vegetación que presentaron la mayor diversidad de endemismo, son el bosque tropical perennifolio (52), el bosque mesófilo de montaña (43), el bosque tropical caducifolio (23), el bosque de Quercus (20), el bosque de coníferas (16), el bosque de galería (15) y el bosque tropical subcaducifolio (11).

Palabras clave: bosque mesófilo de montaña, bosque tropical caducifolio, bosque tropical perennifolio, endemismo, México, Veracruz. 


\section{ABSTRACT}

A list of the endemic taxa of the vascular flora of the state of Veracruz was obtained. We recorded 131 species and 10 subspecific taxa belonging in 56 families and 94 genera. The families with highest number of endemic species were Orchidaceae (11), Myrtaceae (10), Bromeliaceae (9), Poaceae (8), Begoniaceae (7), Zamiaceae (5) y Polypodiaceae (5). The vegetation types that stand out for concentrating the endemic species are: the evergreen tropical forest (52), the cloud forest (43), the deciduous tropical forest (23), the Quercus forest (20), the coniferous forest (16), the gallery forest (15) and the sub-deciduous tropical forest (11).

Key words: cloud forest, endemism, evergreen tropical forest, Mexico, tropical deciduous forest, Veracruz.

\section{INTRODUCCIÓN}

Veracruz se caracteriza por ser uno de los estados más ricos en plantas vasculares, después de Chiapas y Oaxaca (Rzedowski, 1991; 1993). Estimaciones recientes indican que el número de especies de fanerógamas para la flora de Veracruz es aproximadamente de 7490 (Sosa y Gómez-Pompa, 1994), que sumado a las 508 de pteridofitas (Palacios-Rios, 1992; Riba, 1993), hacen un total de 7,998. Algunos grupos de plantas, como las pteridofitas o el género Quercus, tienen gran diversidad a nivel de especie (casi 50\% del total nacional) en Veracruz (Riba, 1993; Nixon, 1993). El estado se caracteriza también por dar cabida al mayor número de los tipos de vegetación registrados para México, según la clasificación de Rzedowski (1978). Por su importancia destacan los bosques tropicales perennifolios, subcaducifolios y caducifolios, palmares, manglares, vegetación de dunas costeras, bosques de Quercus, bosque mesófilo de montaña, bosques de coníferas y los páramos de altura.

Las condiciones topográficas de Veracruz son muy variadas, su territorio se integra con una planicie costera que pertenece a la provincia biogeográfica del Golfo de México y un sistema montañoso que forma parte del Eje Volcánico Transmexicano y de la Sierra Madre Oriental (Morrone, 2001). Las altitudes varían desde el nivel del mar hasta más allá de los 5,000 m, como ocurre en el Pico de Orizaba. En consecuencia, las condiciones climáticas también son muy diversas, presentándose un gradiente que incluye desde los climas cálido húmedos y subhúmedos en los sitios de menor altitud, hasta los fríos y muy fríos como en el Cofre de Perote y en el Pico de Orizaba respectivamente. De los 17 subtipos climáticos registrados para el estado, los cálidos ocupan la mayor superficie. Así se tiene que $31 \%$ pertenece al tipo $\mathrm{Aw}_{2}, 22 \%$ al $\mathrm{Am}, 14 \%$ al $\mathrm{Aw}_{1}$ y $10 \%$ al $\mathrm{Aw}_{0}$ 
de la clasificación de Köppen, y 23\% restante corresponde a los climas semicálidos, templados, fríos y secos (Soto y García, 1989).

Considerando la extraordinaria riqueza biológica y la heterogeneidad medioambiental presentes en el estado de Veracruz, se pueden plantear algunas preguntas de relevancia fitogeográfica referidas a las especies con distribución restringida a los límites del mismo: ¿cuál es la proporción de especies endémicas con respecto al total de plantas vasculares?, ¿cuál es la importancia relativa de estas especies bajo diferentes condiciones ecológicas?.

Partiendo de la consideración de que, si bien los patrones de endemismo son distintos para taxa y para regiones diferentes (Gentry, 1986) y su evaluación depende de la escala espacial considerada, en términos generales podemos decir que el endemismo de la flora vascular en México a nivel de familia y de género, se concentra principalmente en las zonas áridas y en la vegetación xerófila, en tanto que a nivel de especies lo hace en los bosques de coníferas y en los encinares de las altas montañas, en las zonas frías y semihúmedas (Rzedowski, 1993). De los bosques tropicales destaca por concentrar el mayor porcentaje de endemismo en México (40\%) el bosque tropical caducifolio y subcaducifolio (Rzedowski, 1993). La información a nivel de estado o región es escasa. La mayoría de las investigaciones se han enfocado sobre ciertas especies amenazadas y/o endémicas, pertenecientes a diversas familias botánicas o tipos de vegetación (Villaseñor, 1990; Hernández y Godínez, 1994; Rzedowski, 1996; Sosa et al., 1998). Para el caso particular del estado de Veracruz no se cuenta con estudios dirigidos al conocimiento del endemismo a nivel de vegetales, sin embargo, entre los trabajos florísticos que citan algunas especies de tal categoría se tienen los de CastilloCampos (1991), Norstog et al. (1992), Zamora y Castillo-Campos (1997) y Vovides et al. (1997).

El objetivo de este análisis es presentar una primera aproximación al estudio del endemismo en Veracruz mediante la elaboración de una lista preliminar de las especies de plantas vasculares que restringen su distribución a los límites de este estado, incluyendo información relativa a su hábito, usos y los tipos de vegetación donde se desarrollan.

\section{ÁREA DE ESTUDIO}

El estado de Veracruz se localiza en la porción oriental del país, en las inmediaciones del Golfo de México, entre los $17^{\circ} 03^{\prime} 56^{\prime \prime}$ y los $22^{\circ} 27^{\prime} 18^{\prime \prime}$ de latitud norte y los 9336'13" y los 98 36'00" de longitud oeste (Anónimo, 1988; Fig. 1). Comprende una superficie aproximada de $72,410 \mathrm{~km}^{2}$, área que lo sitúa en el décimo lugar con respecto a las demás entidades de la República Mexicana. Sus límites 
son: al norte el estado de Tamaulipas, al este el Golfo de México, al oeste San Luis Potosí, Hidalgo y Puebla, al sur y suroeste Oaxaca y al sureste Chiapas y Tabasco. Presenta una longitud de norte a sur de $780 \mathrm{~km}$, con una amplitud variable entre los 52 y los 212 km (Anónimo, 2001a).

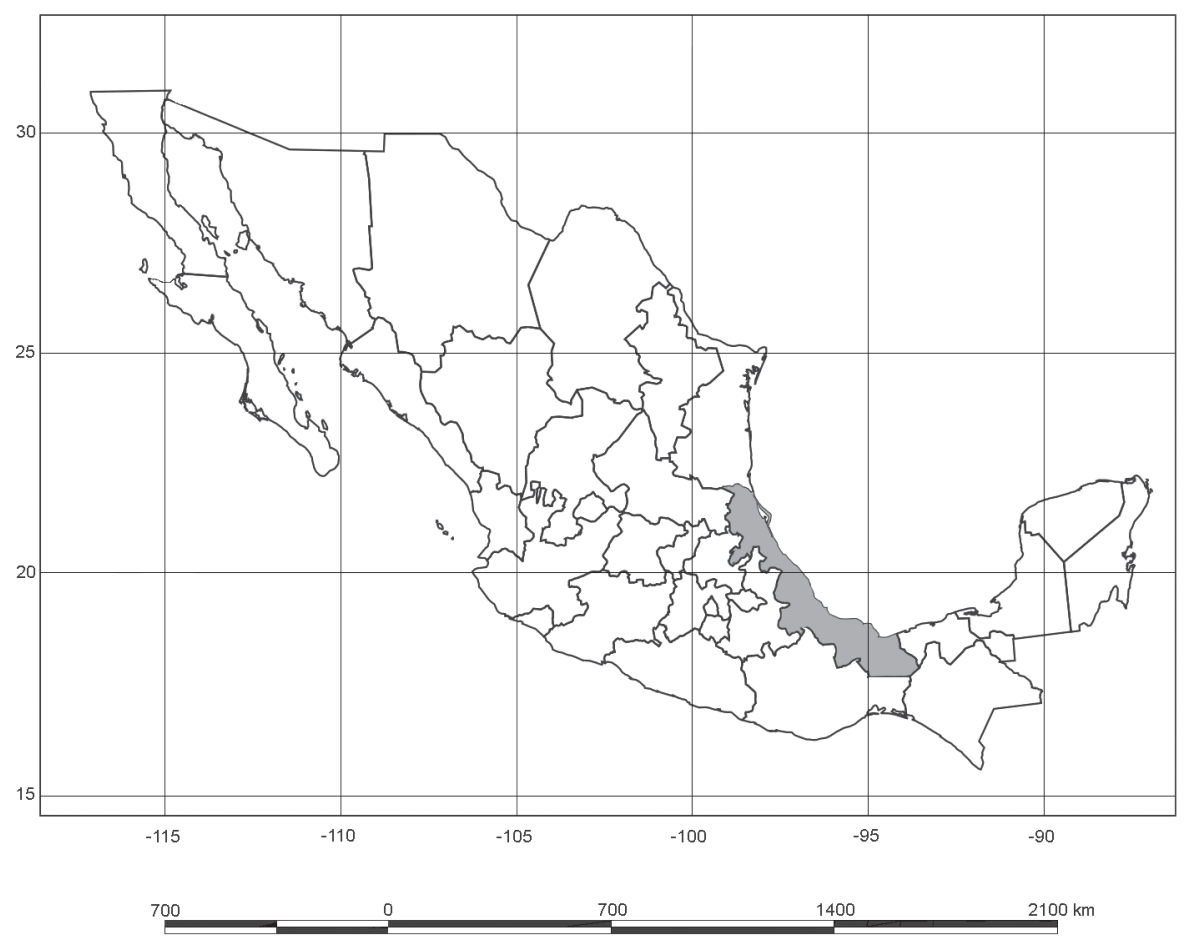

Figura 1. Localización del estado de Veracruz.

\section{MÉTODOS}

Para este trabajo se consideraron como especies endémicas aquellas cuya área de distribución no sobrepasa los límites del territorio veracruzano. Con el fin de integrar la lista se realizó una revisión bibliográfica lo más completa posible de los trabajos florísticos relativos a la entidad, así como de monografías y estudios taxonómicos de familias y géneros que presentan especies en el estado. También se revisaron las colecciones del herbario del Instituto de Ecología, A. C. en Xalapa, Ver. (XAL), las del Herbario Nacional del Instituto de Biología de la Universidad 
Nacional Autónoma de México (MEXU) y las de la Escuela Nacional de Ciencias Biológicas del Instituto Politécnico Nacional (ENCB). Además, se consultaron las bases de datos International Plant Names Index (1999) y Missouri Botanical Garden's VAST (1995).

Entre los trabajos florísticos que registran endemismos de las familias que han sido estudiadas para la Flora de Veracruz, se tienen los de Gregory y Riba (1979), Nash (1979), Nash y Moreno (1981), Gentry (1982a), Espejel (1983), Narave (1983), Vovides (1983, 1994), Nash y Nee (1984), Fernández (1986), Nee (1986, 1993), Sosa et al. (1987), Nevling y Barringer (1988), Sánchez-Vindas (1990), Barringer (1991), Graham (1991), Fryxell (1992), López-Ferrari y EspejoSerna (1993, 1995, 2002), McDonald (1993, 1994), Espejo-Serna y López-Ferrari (1994a, 1998a), Pérez (1995), Rzedowski y Calderón (1996), Durán-Espinoza (1997, 1999), Jiménez y Schubert (1997), Ortega y Ortega (1997), Avendaño (1998), García-Cruz y Sánchez (1999). Las revisiones taxonómicas de diversos grupos de plantas que han aportado información pertinente, son: Bravo-Hollis (1937), Britton y Rose (1963), Hawkes (1965), Smith y Downs (1974, 1979), Gentry (1982b), Soderstrom (1982), Beetle y Miranda (1983), Wunderlin (1983), Lundell (1984a, 1984b, 1984c), McVaugh (1985), Varadarajan y Gilmartin (1988), Luther (1991), Pennington (1997), Croat (1997).

Otros estudios que han proporcionado datos sobre especies endémicas en el estado Veracruz, incluyen los de Hitchcock y Chase (1910), Bravo-Hollis (1970), Handlos (1975), Matuda (1953, 1975, 1976), Balogh (1981), Castillo-Campos y Lorence (1985), Rzedowski y Calderón (1985), Turner (1985, 1988), Sosa y Schubert (1986), Stevenson et al. (1986), García-Franco (1987), Sosa et al. (1987), Lorence y Castillo-Campos (1988), Pérez y Castillo-Campos (1988), Salazar (1988), Castillo-Campos (1991), Espejo-Serna y López-Ferrari (1992, 1993, 1994b, 1995, 1996, 1997a, 1997b, 1998b, 2000a-c), Hietz y Hietz-Seifert (1994), Sosa y GómezPompa (1994), Ibarra-Manríquez y Sinaca (1995), Lascurain (1996), Zamora y Castillo-Campos (1997), Castillo-Campos et al. (1998a), Castillo-Campos y Medina, (1998b), Sosa et al. (1998).

\section{RESULTADOS}

Se registraron 131 especies y 10 taxa subespecíficos endémicos de la flora vascular (Apéndice 1), en total los 141 taxa limitan su distribución geográfica al estado de Veracruz, lo que representa $1.76 \%$ de la flora total registrada para el estado. Este endemismo está repartido en 94 géneros y 56 familias, destacando por el número de taxa las siguientes: Orchidaceae (11), Myrtaceae (10), Bromeliaceae (9), Poaceae (8), Begoniaceae (7), Zamiaceae (5) y Polypodiaceae (5). Las 49 
familias restantes incluyen a los demás componentes (Cuadro 1). De los taxa endémicos $82 \%$ son propios de la vegetación primaria y $10 \%$ de la secundaria, mientras que el $8 \%$ se comparte en ambas comunidades vegetales. En cuanto al hábito de estas plantas, se tiene que $31 \%$ son árboles y arbustos y $69 \%$ corresponde a hierbas y bejucos (Cuadro 2).

Se registraron taxa endémicos en 12 tipos de vegetación, según la clasificación de Rzedowski (1978) (Cuadro 3), entre los cuales destacan los siguientes por presentar el mayor número de taxa endémicos:

Bosque tropical perennifolio

Con 52 taxa endémicos, entre los arbóreos y arbustivos cabe citar a: Antirhea aromatica, Colubrina johnstonii, Eugenia uxpanapensis, Eugenia inirebensis, Eugenia sotoesparzae, Inga lacustris, Inga sinacae y Orthion veracruzense; y de las herbáceas se encuentran Ceratozamia euryphyllidia, Epidendrum dressleri, Olmeca recta, Philodendron subincisum y Stromanthe popolucana.

Bosque mesófilo de montaña

Se registraron 43 taxa endémicos que habitan en este tipo de vegetación, de los cuales cabe citar los siguientes elementos leñosos: Cestrum miradorense, Dichapetalum mexicanum y Rondeletia tuxtlensis. Entre las plantas herbáceas se encuentran Begonia pudica, Impatiens mexicana y Physalis greenmanii.

Bosque tropical caducifolio

Da albergue a 23 taxa endémicos, tanto arbóreos y arbustivos, como Daphnopsis brevifolia, Eugenia ledophylla, Eugenia mozomboensis y Citharexylum kerberi (Nash y Nee, 1984), y también a algunas hierbas, como Begonia polygonata y Zephyranthes miradorensis.

Bosque de Quercus

Cuenta con un número importante de taxa endémicos (20), entre los que destaca Nama linearis, así como Epidendrum stallforthianum, que es de hábito epifítico.

Bosque de coníferas

Dieciseis taxa son endémicos de estos bosques, entre los cuales se encuentra Agave polyacantha var. xalapensis. Otro elemento de distribución restringida, 
Cuadro 1. Familias y géneros que registraron taxa endémicos en el estado de Veracruz, México.

\begin{tabular}{|c|c|c|c|c|c|}
\hline Familia & $\begin{array}{c}\text { Núm. de } \\
\text { taxa }\end{array}$ & $\begin{array}{l}\text { Núm. de } \\
\text { géneros }\end{array}$ & Familia & $\begin{array}{l}\text { Núm. de } \\
\text { taxa }\end{array}$ & $\begin{array}{l}\text { Núm. de } \\
\text { géneros }\end{array}$ \\
\hline Orchidaceae & 11 & 9 & Myrsinaceae & 2 & 1 \\
\hline Myrtaceae & 10 & 2 & Selaginellaceae & 2 & 1 \\
\hline Bromeliaceae & 9 & 3 & Thymelaeaceae & 2 & 1 \\
\hline Poaceae & 8 & 8 & Acanthaceae & 1 & 1 \\
\hline Begoniaceae & 7 & 1 & Alstroemeriaceae & 1 & 1 \\
\hline Polypodiaceae & 5 & 2 & Anthericaceae & 1 & 1 \\
\hline Zamiaceae & 5 & 2 & Arecaceae & 1 & 1 \\
\hline Solanaceae & 4 & 3 & Athyriaceae & 1 & 1 \\
\hline Araceae & 4 & 3 & Balsaminaceae & 1 & 1 \\
\hline Dioscoreaceae & 4 & 1 & Bignoniaceae & 1 & 1 \\
\hline Cactaceae & 3 & 2 & Blechnaceae & 1 & 1 \\
\hline Hydrophyllaceae & 3 & 2 & Bombacaceae & 1 & 1 \\
\hline Fabaceae & 3 & 2 & Boraginaceae & 1 & 1 \\
\hline Agavaceae & 3 & 1 & Burseraceae & 1 & 1 \\
\hline Aristolochiaceae & 3 & 1 & Celastraceae & 1 & 1 \\
\hline Thelypteridaceae & 3 & 1 & Commelinaceae & 1 & 1 \\
\hline Verbenaceae & 3 & 1 & Convallariaceae & 1 & 1 \\
\hline Amaryllidaceae & 2 & 2 & Costaceae & 1 & 1 \\
\hline Asteraceae & 2 & 2 & Dichapetalaceae & 1 & 1 \\
\hline Convolvulaceae & 2 & 2 & Dryopteridaceae & 1 & 1 \\
\hline Cyperaceae & 2 & 2 & Eriocaulaceae & 1 & 1 \\
\hline Hydrangeaceae & 2 & 2 & Iridaceae & 1 & 1 \\
\hline Lomariopsidaceae & 2 & 2 & Juglandaceae & 1 & 1 \\
\hline Malvaceae & 2 & 2 & Lythraceae & 1 & 1 \\
\hline Marantaceae & 2 & 2 & Menispermaceae & 1 & 1 \\
\hline Rhamnaceae & 2 & 2 & Moraceae & 1 & 1 \\
\hline Rubiaceae & 2 & 2 & Smilacaceae & 1 & 1 \\
\hline Violaceae & 2 & 2 & Theophrastaceae & 1 & 1 \\
\hline
\end{tabular}


Cuadro 2. Número de taxa endémicos de Veracruz en cada una de las categorías de hábito.

\begin{tabular}{|l|c|}
\hline Hábito & Núm. de taxa \\
\hline Árboles & 23 \\
\hline Arbustos & 21 \\
\hline Hierbas & 89 \\
\hline Bejucos y lianas & 8 \\
\hline
\end{tabular}

Cuadro 3. Número de taxa endémicos registrados de cada uno de los principales tipos de vegetación del estado de Veracruz, México.

\begin{tabular}{|l|c|}
\hline \multicolumn{1}{|c|}{ Tipo de vegetación } & Núm. de taxa \\
\hline Bosque tropical perennifolio & 52 \\
\hline Bosque mesófilo de montaña & 43 \\
\hline Bosque tropical caducifolio & 23 \\
\hline Bosque de Quercus & 20 \\
\hline Bosque de coníferas & 16 \\
\hline Bosque de galería & 15 \\
\hline Bosque tropical subcaducifolio & 11 \\
\hline Vegetación de dunas costeras & 5 \\
\hline Matorral xerófilo & 3 \\
\hline Pastizal & 2 \\
\hline Palmar & 1 \\
\hline Sabana & 1 \\
\hline Vegetación secundaria & 14 \\
\hline
\end{tabular}

propio de los bosques de coníferas de los sitios perturbados y de los tipos de vegetación colindantes con los pinares, es Echeandia albiflora.

Bosque de galería

Se caracteriza por presentar un número importante de endemismos (15). Entre las especies herbáceas se tiene a Costus dirzoi, al bejuco Hidalgoa uspanapa, a Maianthemum macrophyllum de hábito epifítico y a Ruellia tuxtlensis. 
En los demás tipos de vegetación (Cuadro 3), la riqueza de endemismos varía entre 1 y 11 taxa.

Estado de Conservación

En general los endemitas son propios de la vegetación primaria (Cuadro 3). De los 141 taxa endémicos, 14 se encuentran incluidos en la Norma Oficial Mexicana relativa a la protección ambiental de especies de flora y fauna silvestres nativas de México (Anónimo, 2001b), las cuales tienen diferentes categorías (Cuadro 4). Destacan por encontrarse en peligro de extinción Antirhea aromatica, Ceratozamia euryphyllidia, C. miqueliana, Mammillaria eriacantha, Olmeca recta y Zamia inermis (Anónimo, 2001b; Vovides et al., 1997).

A partir de los datos de los especímenes depositados en los herbarios revisados, algunos autores sugieren que probablemente ciertos taxa considerados de distribución restringida ya se han extinguido. Tal es el caso de Hymenocallis longibracteata, taxon que sólo se conoce de la localidad tipo y aparentemente no se ha vuelto a recolectar desde 1853 (López-Ferrari y Espejo-Serna, 2002). En las mismas circunstancias se registran 30 taxa más (Cuadro 5), que no se han encontrado en las últimas décadas, entre los cuales se pueden citar: Begonia lyniceorum, $B$. polygonata (Jiménez y Schubert, 1997), Evolvulus choapanus, Ipomoea eximia (McDonald, 1993, 1994), Hydrolea ovata var. parvifolia, Nama linearis y $N$. orizabensis (Nash, 1979).

\section{DISCUSIÓN}

Del conjunto aproximado de 7998 taxa de la flora vascular, calculado para el estado de Veracruz, el endemismo de 141 taxa, representa casi 2\% del total. Encontramos que es en la porción central de esta entidad donde se localiza 32\% de elementos de distribución restringida, destacando a este respecto los sitios con bosque mesófilo de montaña y el bosque tropical caducifolio, que albergan el mayor número de ellos en la región. Desafortunadamente, estas comunidades vegetales también son las más amenazadas por el cambio de uso del suelo. En muestreos recientes, en el bosque tropical caducifolio primario, en una superficie de 1.2 ha, se registró $27 \%$ de los 23 taxa endémicos encontrados en este tipo de vegetación en el centro del territorio veracruzano. Lo anterior indica la importancia que tienen los fragmentos de vegetación original que aún persisten en porciones inaccesibles, como la Sierra de Manuel Díaz, áreas que deben tener prioridad para su protección y conservación, ya que representan lo poco que queda del bosque tropical caducifolio. 
Cuadro 4. Especies y entidades subespecíficas endémicas del estado de Veracruz incluidas en la Norma Oficial Mexicana relativa a la protección ambiental de especies de flora y fauna silvestres nativas de México. Simbología de las categorías de vulnerabilidad utilizadas: A = amenazada, $\mathrm{P}=$ en peligro de extinción y $\operatorname{Pr}=$ sujeta a protección especial.

\begin{tabular}{|l|c|l|}
\hline Taxa & Categoría & Fuentes \\
\hline Alfaroa mexicana & $\mathrm{Pr}$ & Anónimo (2001) \\
\hline Antirhea aromatica & $\mathrm{P}$ & Vovides et al. (1997) \\
\hline Bauhinia jucunda & $\mathrm{A}$ & Vovides et al. (1997) \\
\hline Ceratozamia euryphyllidia & $\mathrm{P}$ & Anónimo (2001), Vovides et al. (1997) \\
\hline Ceratozamia mexicana var. latifolia & $\mathrm{A}$ & Anónimo (2001), Vovides et al. (1997) \\
\hline Ceratozamia miqueliana & $\mathrm{P}$ & Anónimo (2001), Vovides et al. (1997) \\
\hline Coryphantha greenwoodii & $\mathrm{Pr}$ & Anónimo (2001) \\
\hline Epidendrum dressleri & $\mathrm{Pr}$ & Anónimo (2001) \\
\hline Mammillaria eriacantha & $\mathrm{P}$ & Vovides et al. (1997) \\
\hline Mammillaria sartorii & $\mathrm{A}$ & Vovides et al. (1997) \\
\hline Olmeca recta & $\mathrm{P}$ & Anónimo (2001) \\
\hline Oncidium stramineum & $\mathrm{A}$ & Anónimo (2001), Vovides et al. (1997) \\
\hline Zamia furfuracea & $\mathrm{A}$ & Anónimo (2001), Vovides et al. (1997) \\
\hline Zamia inermis & $\mathrm{P}$ & Anónimo (2001), Vovides et al. (1997) \\
\hline
\end{tabular}

Aproximadamente $16 \%$ de 141 taxa de la flora endémica registrada hasta el momento corresponde a árboles, lo que representa 1\% de las 2,300 especies arbóreas que actualmente se estiman para el estado de Veracruz.

Es difícil determinar el riesgo de extinción de las especies, sin embargo, por la rareza que presentan 14 de los taxa incluidos en este estudio y la modificación del hábitat en donde se han registrado, se considera que probablemente éstos, deberían ser incluidos en la Norma Oficial Mexicana (Anónimo, 2001b), como especies que se encuentran en peligro de extinción o extintas. Sobre todo de aquellas especies que se han registrado una sola vez y de una localidad, la cual en la actualidad se encuentra totalmente modificada y cuya vegetación original ha sido sustituida por cultivos y pastizales, como es el caso de Hymenocallis longibracteata (Cuadro 5).

Como puede verse en los resultados de este estudio, los taxa endémicos se han registrado primordialmente en la vegetación primaria y muy pocos $(8 \%)$ se han encontrado tanto en la primaria como en la secundaria. Lo anterior sugiere una alta 
Cuadro 5. Especies y entidades subespecíficas endémicas de Veracruz conocidas de una sola colecta.

\begin{tabular}{|c|c|}
\hline \multicolumn{2}{|c|}{ Especie } \\
\hline Agave ellemeetiana Jacobi & Hydrolea ovata var. parvifolia D. Nash \\
\hline Aristida fournieriana Hitchc. & Hymenocallis longibracteata Hochr. \\
\hline Axonopus multipes Swallen & Impatiens mexicana $\mathrm{Rydb}$. \\
\hline Begonia lyniceorum Burt-Utley & Ipomoea eximia House \\
\hline Begonia polygonata Liebm. & Mammillaria eriacantha Link \& Otto \\
\hline Citharexylum fulgidum Moldenke & Mammillaria sartorii J. A. Purpus \\
\hline Coryphantha greenwoodii Bravo & Mormodes tuxtlensis Salazar \\
\hline Dichapetalum mexicanum Prance & Muhlenbergia laxa Hitchc. \\
\hline Dioscorea cruzensis R. Knuth & Nama linearis D. Nash \\
\hline Dioscorea orizabensis Uline ex Knuth & Nama orizabensis D. Nash \\
\hline Dioscorea spiculoides Matuda & Olmeca recta Soderstr. \\
\hline Eugenia mozomboensis P. E. Sánchez & Orthion veracruzense Lundell \\
\hline Euonymus platyphyllus Lundell & Parathesis tuxtlensis Lundell \\
\hline Evolvulus choapanus McDonald & Rinorea uxpanapana $\mathrm{T}$. Wendt \\
\hline Hechtia glabra Brandegee & Tillandsia flavobracteata Matuda \\
\hline Hechtia lindmanioides L. B. Sm. & \\
\hline
\end{tabular}

especificidad del hábitat de las plantas en cuestión y su vulnerabilidad a la perturbación.

Es difícil detener la degradación de la vegetación primaria, sin embargo, si no se toman las medidas de conservación correspondientes, las especies endémicas podrían seguir el camino de la extinción en tiempos relativamente cortos, considerando que la superficie de vegetación original que aún queda en el estado de Veracruz es muy pequeña (aproximadamente 10\% del territorio de la entidad, según Guzmán y Castillo-Campos (1989)).

La riqueza de plantas endémicas del estado de Veracruz se encuentra amenazada debido a la ampliación de la frontera agrícola y ganadera, misma que ha ocasionado que la vegetación original vaya desapareciendo cada día a un ritmo alarmante. Aunque estudios recientes (Harte y Kinzig, 1997; Kinzig y Harte, 2000) indican que la pérdida de especies endémicas por destrucción o transformación del hábitat puede ser menor que la estimada a partir de la relación área-especie de la teoría de biogeografía de islas (May et al., 1995); los cambios en el uso del suelo 
conducen a una degradación de la calidad del hábitat por la fragmentación, el efecto de borde y la pérdida de corredores biológicos (Kinzig y Harte, 2000). También se debe considerar peligrosa la extracción selectiva de especies útiles, la invasión de especies exóticas, la deposición de contaminantes e incluso los efectos del cambio climático global (Heywood y Watson, 1995). La mayoría de las especies endémicas son altamente sensibles a la transformación de la cobertura vegetal primaria, así como a las modificaciones que se presentan en las condiciones ecológicas que prevalecen en esos hábitats.

Finalmente, queremos enfatizar que el estudio del endemismo en el estado de Veracruz en particular y de la República Mexicana en general, es todavía un expediente abierto. Seguramente el registro de especies de área restringida se incrementará a medida que se avance en el conocimiento de la flora de Veracruz. A partir de tal inventario se deberán abordar problemas relacionados con las causas e implicaciones ecológicas y evolutivas, así como aquellos relacionados con la conservación y manejo de las especies endémicas.

\section{AGRADECIMIENTOS}

Deseamos agradecer a la Dra. Luciana Porter por la traducción del resumen al idioma inglés. A la Dra. María Luisa Martínez y al M. en C. Sergio Avendaño Reyes por la revisión del manuscrito y de manera especial al revisor anónimo del comité editorial de Acta Botanica Mexicana por sus atinadas sugerencias. Este trabajo fue realizado con financiamiento del Instituto de Ecología, A. C., a través del Departamento de Sistemática Vegetal (902-14) y forma parte de la tesis doctoral del primer autor, en el Programa de Posgrado en Ciencias Biológicas de la Universidad Autónoma Metropolitana Unidad-Iztapalapa.

\section{LITERATURA CITADA}

Anónimo. 1988. Síntesis geográfica, nomenclátor y anexo cartográfico del estado de Veracruz. Instituto Nacional de Estadística, Geografía e Informática. Aguascalientes, Ags. 69 pp.

Anónimo. 2001a. Sistema de información ambiental de Veracruz. Subsecretaría de Medio Ambiente-Secretaría de Desarrollo Regional-Gobierno del Estado de Veracruz. Xalapa, Ver. 23 pp.

Anónimo. 2001b. Norma Oficial Mexicana NOM-059-ECOL-2001. Instituto Nacional de Ecología-Secretaría de Medio Ambiente y Recursos Naturales. México, D.F. 88 pp.

Avendaño, S. 1998. Bombacaceae. Flora de Veracruz. Fascículo 107. Instituto de Ecología, A.C.-University of California. Xalapa, Ver. 40 pp. 
Balogh, P. 1981. Nomenclatural notes on the genus Schiedeella Schlechter (Orchidaceae). Orquídea (Méx.) 8(1): 38-40.

Barringer, K. 1991. Balsaminaceae. Flora de Veracruz. Fascículo 64. Instituto de Ecología, A.C.-University of California. Xalapa, Ver. 8 pp.

Beetle, A. A. y J. A. Miranda S. 1983. Las gramíneas de México. Tomo I. Secretaría de Agricultura y Recursos Hidraúlicos. México, D.F. 260 pp.

Beetle, A. A., J. A. Miranda S., V. Jaramillo L., A. M. Rodríguez R., L. Aragón M., M. A. Vergara B., A. Chimal H., O. Domínguez S. 1995. Las gramíneas de México. Tomo IV. Secretaría de Agricultura, Ganadería y Desarrollo Rural. México, D.F. 342 pp.

Bravo-Hollis, H. 1937. Las cactáceas de México. Universidad Nacional Autónoma de México. México, D.F. 755 pp.

Bravo-Hollis, H. 1970. Una especie nueva del género Coryphantha. Cact. Suc. Mex. 15(2): 27.

Britton, N. L. y J. N. Rose. 1963. The Cactaceae. Vol. IV. Dover Publications. Nueva York. $318 \mathrm{pp}$.

Castillo-Campos, G. y D. H. Lorence. 1985. Antirhea aromatica (Rubiaceae, Guettardeae), a new species from Veracruz, Mexico. Ann. Missouri Bot. Gard. 72: 268-271.

Castillo-Campos, G. 1991. Vegetación y flora del municipio de Xalapa. Programa del Hombre y la Biosfera (MAB, UNESCO), Instituto de Ecología, A.C., H. Ayuntamiento de Xalapa, Veracruz. Xalapa, Ver. 148 pp.

Castillo-Campos, G., A. P. Vovides y M. Vázquez T. 1998a. Una nueva especie de Stromanthe (Marantaceae) de Veracruz, México. Polibotánica 8: 13-19.

Castillo-Campos, G. y Ma. E. Medina. 1998b. A new species of Jacquinia (Theophrastaceae) from Veracruz, Mexico. Novon 8(2): 129-132.

Croat, T. B. 1997. A revision of Philodendron subgenus Philodendron (Araceae) for Mexico and Central America. Ann. Missouri Bot. Gard. 84(3): 311-704.

Cronquist, A. 1988. The evolution and classification of flowering plants. The New York Botanical Garden. Nueva York. 555 pp.

Durán-Espinosa, C. 1997. Dichapetalaceae. Flora de Veracruz. Fascículo 101. Instituto de Ecología, A.C.-University of California. Xalapa, Ver. 10 pp.

Durán-Espinosa, C. 1999. Hydrangeaceae. Flora de Veracruz. Fascículo 109. Instituto de Ecología, A.C.-University of California. Xalapa, Ver. 22 pp.

Espejel, I. 1983. Garryaceae. Flora de Veracruz. Fascículo 33. Instituto Nacional de Investigaciones sobre Recursos Bióticos. Xalapa, Ver. 6 pp.

Espejo-Serna, A. y A. R. López-Ferrari. 1992. Las monocotiledóneas mexicanas. Una sinopsis florística. Parte I. Consejo Nacional de la Flora de México-Universidad Autónoma Metropolitana. México, D.F. 76 pp.

Espejo-Serna, A. y A. R. López-Ferrari. 1993. Las monocotiledóneas mexicanas. Una sinopsis florística. Parte II. Consejo Nacional de la Flora de México-Universidad Autónoma Metropolitana. México, D.F. 70 pp.

Espejo-Serna, A. y A. R. López-Ferrari. 1994a. Alstroemeriaceae. Flora de Veracruz. Fascículo 83. Instituto de Ecología, A.C.-University of California. Xalapa, Ver. 12 pp. 
Espejo-Serna, A. y A. R. López-Ferrari. 1994b. Las monocotiledóneas mexicanas. Una sinopsis florística. Parte III. Consejo Nacional de la Flora de México-Universidad Autónoma Metropolitana. México, D.F. 73 pp.

Espejo-Serna, A. y A. R. López-Ferrari. 1995. Las monocotiledóneas mexicanas. Una sinopsis florística. Parte IV. Consejo Nacional de la Flora de México-Universidad Autónoma Metropolitana. México, D.F. 49 pp.

Espejo-Serna, A. y A. R. López-Ferrari. 1996. Las monocotiledóneas mexicanas. Una sinopsis florística. Parte VI. Consejo Nacional de la Flora de México-Universidad Autónoma Metropolitana. México, D.F. 116 pp.

Espejo-Serna, A. y A. R. López-Ferrari. 1997a. Las monocotiledóneas mexicanas. Una sinopsis florística. Parte V. Consejo Nacional de la Flora de México-Universidad Autónoma Metropolitana. México, D.F. 98 pp.

Espejo-Serna, A. y A. R. López-Ferrari. 1997b. Las monocotiledóneas mexicanas. Una sinopsis florística. Parte VII. Consejo Nacional de la Flora de México-Universidad Autónoma Metropolitana. México, D.F. 90 pp.

Espejo-Serna, A. y A. R. López-Ferrari. 1998a. Iridaceae. Flora de Veracruz. Fascículo 105. Instituto de Ecología, A.C.-University of California. Xalapa, Ver. 58 pp.

Espejo-Serna, A. y A. R. López-Ferrari. 1998b. Las monocotiledóneas mexicanas. Una sinopsis florística. Parte VIII. Consejo Nacional de la Flora de México-Universidad Autónoma Metropolitana. México, D.F. 115 pp.

Espejo-Serna, A. y A. R. López-Ferrari. 2000a-c. Las monocotiledóneas mexicanas. Una sinopsis florística. Partes IX-XI. Consejo Nacional de la Flora de México-Universidad Autónoma Metropolitana. México, D.F. 337 pp.

Fernández, R. 1986. Rhamnaceae. Flora de Veracruz. Fascículo 50. Instituto Nacional de Investigaciones sobre Recursos Bióticos. Xalapa, Ver. 63 pp.

Fryxell, P. A. 1992. Malvaceae. Flora de Veracruz. Fascículo 68. Instituto de Ecología, A.C.University of California. Xalapa, Ver. 255 pp.

García-Cruz, J. y L. Sánchez S. 1999. Orchidaceae II. Epidendrum. Flora de Veracruz. Fascículo 112. Instituto de Ecología, A.C.-University of California. Xalapa, Ver. 110 pp.

García-Franco, J. G. 1987. Las Bromelias de México. Instituto Nacional de Investigaciones sobre Recursos Bióticos. Xalapa, Ver. 94 pp.

Gentry, A. H. 1982a. Bignoniaceae. Flora de Veracruz. Fascículo 24. Instituto Nacional de Investigaciones sobre Recursos Bióticos. Xalapa, Ver. 222 pp.

Gentry, A. H. 1986. Endemism in tropical versus temperate plant communities. In: Soulé, M. E. (ed.). Conservation biology. The science of scarcity and diversity. Sinauer. Sunderland, Massachusetts. pp.153-181.

Gentry, H. S. 1982b. Agaves of continental North America. The University of Arizona Press. Tucson, Arizona. 670 pp.

Graham, S. A. 1991. Lythraceae. Flora de Veracruz. Fascículo 66. Instituto de Ecología, A.C.-University of California. Xalapa, Ver. $94 \mathrm{pp}$.

Gregory, D. y R. Riba. 1979. Selaginellaceae. Flora de Veracruz. Fascículo 6. Instituto Nacional de Investigaciones sobre Recursos Bióticos. Xalapa, Ver. 35 pp. 
Guzmán, G. S. y G. Castillo-Campos. 1989. Uso del suelo en Veracruz. Extensión 32: 3135.

Handlos, W. L. 1975. The taxonomy of Tripogandra (Commelinaceae). Rhodora 77: 213333.

Harte, J. y A. P. Kinzig. 1997. On the implications of species-area relationships for endemism, spatial turnover, and food web patterns. Oikos 80: 417-427.

Hawkes, A. D. 1965. Encyclopaedia of cultivated orchids. Faber and Faber Limited. Londres. $602 \mathrm{pp}$.

Hernández, H. M. y A. Godínez. 1994. Contribución al conocimiento de las cactáceas mexicanas amenazadas. Acta Bot. Mex. 26: 33-52.

Heywood, V. H. y R. T. Watson. 1995. Global diversity assessment. Cambridge University Press. Cambridge. 1140 pp.

Hietz, P. y U. Hietz-Seifert. 1994. Epífitas de Veracruz. Instituto de Ecología, A.C. Xalapa, Ver. $236 \mathrm{pp}$.

Hitchcock, A. S. y A. Chase. 1910. The North American species of Panicum. Contr. U. S. Natl. Herb. 15: 1-331.

Ibarra-Manríquez, G. y S. Sinaca C. 1995. Lista florística comentada de la Estación de Biología Tropical "Los Tuxtlas", Veracruz, México. Rev. Biol. Trop. 43(1-3): 75115.

International Plant Names Index (IPNI). 1999. Published on the Internet http://www.ipni.org. (Accessed 2002).

Jiménez, R. y B. G. Schubert. 1997. Begoniaceae. Flora de Veracruz. Fascículo 100. Instituto de Ecología, A.C.-University of California. Xalapa, Ver. 70 pp.

Kinzig, A. P. y J. Harte. 2000. Implications of endemics-area relationships for estimates of species extinctions. Ecology 81: 3305-3311.

Lascurain, M. 1996. A new species of Calathea (Marantaceae) from Veracruz, Mexico. Novon 6: 385-388.

López-Ferrari, A. R. y A. Espejo-Serna. 1993. Convallariaceae. Flora de Veracruz. Fascículo 76. Instituto de Ecología, A.C.-University of California. Xalapa, Ver. 20 pp.

López-Ferrari, A. R. y A. Espejo-Serna. 1995. Anthericaceae. Flora de Veracruz. Fascículo 86. Instituto de Ecología, A.C.-University of California. Xalapa, Ver. 20 pp.

López-Ferrari, A. R. y A. Espejo-Serna. 2002. Amaryllidaceae. Flora de Veracruz. Fascículo 128. Instituto de Ecología, A.C.-University of California. Xalapa, Ver. 32 pp.

Lorence, D. H. y G. Castillo-Campos. 1988. Tres nuevas especies y una nueva combinación en el género Rondeletia (Rubiaceae, Rondeletieae) de Veracruz y Oaxaca, México. Biótica 13(2): 147-157.

Lundell, C. L. 1984a. Neotropical Myrsinaceae-XIII. Phytologia 56(3): 141-143.

Lundell, C. L. 1984b. Studies of American plants-XXI. Phytologia 56(1): 28-31.

Lundell, C. L. 1984c. A new species of Euonymus (Celastraceae) from Mexico. Phytologia 56(6): 419.

Luther, H. 1991. Miscellaneous new taxa of Bromeliaceae (VIII). Selbyana 12: 68-90.

Matuda, E. 1953. Nuevas dioscóreas de México y Guatemala. Anales Inst. Biol. Univ. Nac. Autón. México 24: 55-61.

Matuda, E. 1975. Tres nuevas especies de Tillandsia. Cact. Suc. Mex. 20(4): 96-99. 
Matuda, E. 1976. Tres nuevas especies de plantas mexicanas. Cact. Suc. Mex. 21(3): 7476.

May, R. M., J. H. Lawton y N. E. Store. 1995. Assessing extinction rates. In: Lawton, J. H. y R. M. May (eds.). Extinction rates. Oxford University Press. Nueva York. pp. 1-24.

McDonald, A. 1993. Convolvulaceae I. Flora de Veracruz. Fascículo 73. Instituto de Ecología, A.C.-University of California. Xalapa, Ver. 99 pp.

McDonald, A. 1994. Convolvulaceae II. Flora de Veracruz. Fascículo 77. Instituto de Ecología, A.C.-University of California. Xalapa, Ver. 133 pp.

McVaugh, R. 1985. Orchidaceae. Flora Novo-Galiciana 14: 1-363.

Missouri Botanical Garden's VAST. 1995. (VAScular Tropicos) nomenclatural database and associated authority files. Published on the Internet http://www.mobot.org. (Accessed 2002).

Morrone, J. J. 2001. Biogeografía de América Latina y el Caribe. M\&T-Manuales \& Tesis SEA, Vol. III. Zaragoza. 148 pp.

Narave, H. 1983. Juglandaceae. Flora de Veracruz. Fascículo 31. Instituto Nacional de Investigaciones sobre Recursos Bióticos. Xalapa, Ver. 30 pp.

Nash, D. L. 1979 Hydrophyllaceae. Flora de Veracruz. Fascículo 5. Instituto Nacional de Investigaciones sobre Recursos Bióticos. Xalapa, Ver. 37 pp.

Nash, D. L. y N. P. Moreno. 1981. Boraginaceae. Flora de Veracruz. Fascículo 18. Instituto Nacional de Investigaciones sobre Recursos Bióticos. Xalapa, Ver. 149 pp.

Nash, D. L. y M. Nee. 1984. Verbenaceae. Flora de Veracruz. Fascículo 41. Instituto Nacional de Investigaciones sobre Recursos Bióticos. Xalapa, Ver. 154 pp.

Nee, M. 1986. Solanaceae I. Flora de Veracruz. Fascículo 49. Instituto Nacional de Investigaciones sobre Recursos Bióticos. Xalapa, Ver. 191 pp.

Nee, M. 1993. Solanaceae II. Flora de Veracruz. Fascículo 72. Instituto de Ecología, A.C.University of California. Xalapa, Ver. $158 \mathrm{pp}$.

Nevling, L. I. y K. Barringer. 1988. Thymelaeaceae. Flora de Veracruz. Fascículo 59. Instituto Nacional de Investigaciones sobre Recursos Bióticos. Xalapa, Ver. 16 pp.

Nixon, K. C. 1993. The genus Quercus in Mexico. In: Ramamoorthy, T. P., Bye, R., Lot, A., Fa, J. (eds.). Biological diversity of Mexico: Origins and distribution. Oxford University Press. Nueva York. pp 447-458.

Norstog, K. J., P. K. S. Fawcett y A. P. Vovides. 1992. Beetle pollination of two species of Zamia: evolutionary and ecological considerations. Paleobotanist 41: 149-158.

Ortega, J. F. y R. V. Ortega O. 1997. Aristolochiaceae. Flora de Veracruz. Fascículo 99. Instituto de Ecología, A.C.-University of California. Xalapa, Ver. 46 pp.

Palacios-Rios, M. 1992. Las pteridofitas del estado de Veracruz, México. Tesis de Maestría. Facultad de Ciencias, Universidad Nacional Autónoma de México. México, D.F. 364 pp.

Pennington, T. D. 1997. The genus Inga. Botany. The Royal Botanic Gardens, Kew. 844 pp.

Pérez, E. 1995. Menispermaceae. Flora de Veracruz. Fascículo 87. Instituto de Ecología, A.C.-University of California. Xalapa, Ver. 43 pp. 
Pérez, E. y G. Castillo-Campos 1988. Una nueva especie de Hyperbaena (Menispermaceae) de Veracruz, México. Acta Bot. Mex. 4: 15-19.

Riba, R. 1993. Mexican pteridophytes: Distribution and endemism. In: Ramamoorthy, T. P., Bye, R., Lot, A., Fa, J. (eds.). Biological diversity of Mexico: Origins and distribution. Oxford University Press. Nueva York. pp. 379-395.

Rzedowski, J. 1978. Vegetación de México. Editorial Limusa. México, D.F. 432 pp.

Rzedowski, J. 1991. Diversidad y orígenes de la flora fanerogámica de México. Acta Bot. Mex. 14: 3-21.

Rzedowski, J. 1993. Diversity and origins of the phanerogamic flora of Mexico. In: Ramamoorthy, T. P., Bye, R., Lot, A., Fa, J. (eds.). Biological diversity of Mexico: Origins and distribution. Oxford University Press. Nueva York. pp. 129-144.

Rzedowski, J. 1996. Análisis preliminar de la flora vascular de los bosques mesófilos de montaña de México. Acta Bot. Mex. 35: 25-44.

Rzedowski, J. y G. Calderón de Rzedowski. 1985. Flora fanerogámica del Valle de México. Vol. II. Instituto de Ecología, A.C. 674 pp.

Rzedowski, J. y G. Calderón de Rzedowski. 1996. Burseraceae. Flora de Veracruz. Fascículo 94. Instituto de Ecología, A.C.-University of California. Xalapa, Ver. 37 pp.

Salazar, G. A. 1988. Mormodes tuxtlensis, a new species from Veracruz, Mexico. Orquídea (Mex.) 11: 59-62.

Sánchez-Vindas, P. E. 1990. Myrtaceae. Flora de Veracruz. Fascículo 62. Instituto de Ecología, A.C.-University of California. Xalapa, Ver. 146 pp.

Smith, L. y R. J. Downs. 1974. Pitcairnioideae (Bromeliaceae). Monograph No. 14. Part 1. Flora Neotropica. Hafner Press. Nueva York. pp. 1-658.

Smith, L. y R. J. Downs. 1979. Bromelioideae (Bromeliaceae). Monograph No. 14. Part 3. Flora Neotropica. Hafner Press. Nueva York. pp. 1493-2142.

Soderstrom, T. R. 1982. Validation of the generic name Olmeca and its two species (Poaceae: Bambusoideae). Phytologia 51(2): 161.

Sosa, V. y B. G. Schubert. 1986. Una nueva variedad de Dioscorea spiculiflora Hemsl. (Dioscoreaceae) de Veracruz, México. Biótica 11(3): 187-190.

Sosa, V., B. G. Schubert y A. Gómez-Pompa. 1987. Dioscoreaceae. Flora de Veracruz. Fascículo 53. Instituto Nacional de Investigaciones sobre Recursos Bióticos. Xalapa, Ver. 46 pp.

Sosa, V. y A. Gómez-Pompa (comp.) 1994. Lista florística. Flora de Veracruz. Fascículo 82. Instituto de Ecología, A.C.-University of California. Xalapa, Ver. 245 pp.

Sosa, V., A. P. Vovides y G. Castillo-Campos. 1998. Monitoring endemic plant extinction in Veracruz, Mexico. Biodiversity and Conservation 7: 1521-1527.

Soto, M. y E. García. 1989. Atlas climático del estado de Veracruz. Instituto de Ecología, A.C. Xalapa, Ver. 125 pp.

Stevenson, D. W., S. Sabato y M. Vázquez-Torres. 1986. A new species of Ceratozamia (Zamiaceae) from Veracruz, Mexico with comments on species relationships, habitats, and vegetative morphology in Ceratozamia. Brittonia 38(1): 17-26.

Turner, B. L. 1985. A new species of Neurolaena (Asteraceae-Heliantheae) from southernmost Veracruz, México. Phytologia 58(7): 497. 
Turner, B. L. 1988. A new species of Hidalgoa (Asteraceae,Coreopsideae) from southern Mexico. Phytologia 65(5): 379-381.

Varadarajan, G. S. y A. J. Gilmartin. 1988. Taxonomic realignments within the subfamily Pitcairnioideae (Bromeliaceae). Syst. Bot. 13(2): 294-299.

Villaseñor, J. L. 1990. The genera of Asteraceae endemic to Mexico and adjacent regions. Aliso 12(4): 685-692.

Vovides, A. 1983. Zamiaceae. Flora de Veracruz. Fascículo 26. Instituto Nacional de Investigaciones sobre Recursos Bióticos. Xalapa, Ver. $31 \mathrm{pp}$.

Vovides, A. 1994. Costaceae. Flora de Veracruz. Fascículo 78. Instituto de Ecología, A.C.University of California. Xalapa, Ver. 13 pp.

Vovides, A. P., V. Luna y G. Medina. 1997. Relación de algunas plantas y hongos mexicanos raros, amenazados o en peligro de extinción y sugerencias para su conservación. Acta Bot. Mex. 39: 1-42.

Wendt, T. 1983. Plantae Uxpanapae II. Novedades en Violaceae y Scrophulariaceae. Bol. Soc. Bot. Méx. 45: 133-140.

Wunderlin, R. P. 1983. Revision of arborescent bauhinias (Fabaceae: Caesalpinioideae: Cercideae) native to middle America. Ann. Missouri Bot. Gard. 70: 95-127.

Zamora, C. P. y G. Castillo-Campos. 1997. Vegetación y flora del municipio de Tlalnelhuayocan, Veracruz. Textos Universitarios. Universidad Veracruzana. Xalapa, Ver. $88 \mathrm{pp}$. 
Apéndice 1. Lista de los taxa endémicos registrados para el estado de Veracruz, agrupados siguiendo la propuesta de clasificación de Cronquist (1988). Se incluye, dependiendo de la información disponible, el nombre válido, nombre común, hábito, tipo de vegetación, uso, comentarios y fuentes de información. Para diferenciar el hábito de las especies se utilizaron los términos más comunes (árbol, arbusto, hierba, bejuco), incluyendo a las lianas en los bejucos.

\section{PTERIDOPHYTA}

\section{ATHYRIACEAE}

Diplazium hahnii (Fourn.) C. Chr.

Hábito: hierba.

Tipo de vegetación: bosque mesófilo de montaña.

Fuente: Palacios-Rios, 1992.

\section{BLECHNACEAE}

Blechnum danaeaceum (Kunze) C. Chr.

Hábito: hierba.

Tipos de vegetación: bosque mesófilo de montaña, bosque de coníferas.

Fuente: Palacios-Rios, 1992.

\section{DRYOPTERIDACEAE}

Polystichum ordinatum $x$ muricatum

Hábito: hierba.

Tipo de vegetación: bosque mesófilo de montaña.

Fuente: Palacios-Rios, 1992.

\section{LOMARIOPSIDACEAE}

Bolbitis umbrosa (Liebm.) Ching

Hábito: hierba.

Tipo de vegetación: bosque tropical perennifolio.

Fuentes: Palacios-Rios, 1992; Riba, 1993.

Elaphoglossum obscurum (Fourn.) C. Chr.

Hábito: hierba.

Tipos de vegetación: bosque de Quercus, bosque mesófilo de montaña, bosque de coníferas.

Fuente: Palacios-Rios, 1992.

\section{POLYPODIACEAE}

Pleopeltis x melanoneuron Mickel \& Beitel

Hábito: hierba.

Tipo de vegetación: bosque mesófilo de montaña.

Fuente: Palacios-Rios, 1992. 
Apéndice. Continuación.

Pleopeltis x sordidula (Maxon \& Weatherby) Mickel \& Beitel.

Hábito: hierba.

Tipos de vegetación: bosque mesófilo de montaña, bosque tropical perennifolio.

Fuente: Palacios-Rios, 1992.

Polypodium arthropodium Fée

Hábito: hierba.

Tipos de vegetación: bosque de Quercus, bosque tropical perennifolio.

Fuente: Palacios-Rios, 1992.

Polypodium hahnii Fourn.

Hábito: hierba.

Tipo de vegetación: bosque mesófilo de montaña.

Fuente: Palacios-Rios, 1992.

Polypodium lesourdianum Fourn.

Hábito: hierba.

Tipo de vegetación: bosque mesófilo de montaña.

Fuente: Palacios-Rios, 1992; Riba, 1993.

SELAGINELLACEAE

Selaginella pulcherrima Liebm. ex Fourn.

Hábito: hierba.

Tipos de vegetación: bosque tropical perennifolio, bosque mesófilo de montaña.

Fuente: Gregory y Riba, 1979.

Selaginella orizabensis Hieron.

Hábito: hierba.

Tipo de vegetación: bosque mesófilo de montaña.

Fuente: Palacios-Rios, 1992.

THELYPTERIDACEAE

Thelypteris lanosa (C. Chr.) A. R. Smith

Hábito: hierba.

Tipos de vegetación: bosque mesófilo de montaña, bosque de Quercus.

Fuente: Palacios-Rios, 1992.

Thelypteris schaffneri (Fée) Reed

Hábito: hierba. 
Apéndice. Continuación.

Tipo de vegetación: bosque mesófilo de montaña.

Fuente: Palacios-Rios, 1992.

Thelypteris rhachiflexuosa Riba

Hábito: hierba.

Tipo de vegetación: bosque tropical perennifolio.

Fuente: Palacios-Rios, 1992.

\section{GYMNOSPERMAE}

\section{ZAMIACEAE}

Ceratozamia euryphyllidia Vázquez-Torres, Sabato \& D. Stevenson

Hábito: hierba.

Tipo de vegetación: bosque tropical perennifolio.

Fuente: Stevenson et al., 1986.

Ceratozamia mexicana Brongn. var. latifolia (Miq.) Schuster

Hábito: hierba.

Tipo de vegetación: bosque mesófilo de montaña.

Fuente: Vovides, 1983.

Ceratozamia miqueliana Wendl.

Nombre común: palmita (sur de Veracruz).

Hábito: hierba.

Tipo de vegetación: bosque tropical perennifolio.

Uso: ornamental.

Fuente: Vovides, 1983.

Zamia furfuracea $\mathrm{L}$. f.

Nombre común: helecho marino.

Hábito: hierba.

Tipos de vegetación: dunas costeras, palmar, bosque tropical perennifolio, bosque tropical caducifolio, bosque tropical subcaducifolio.

Uso: ornamental.

Fuente: Vovides, 1983.

Zamia inermis Vovides, Rees \& Vázquez-Torres

Hábito: hierba.

Tipo de vegetación: bosque tropical caducifolio.

Fuente: Vovides, 1983. 
Apéndice. Continuación.

ANGIOSPERMAE

MONOCOTYLEDONEAE

AGAVACEAE

Agave ellemeetiana Jacobi

Hábito: hierba.

Fuentes: Gentry, 1982 b.; Espejo-Serna y López-Ferrari, 1992.

Agave horrida Lemaire ex Jacobi subsp. perotensis Ullrich

Nombre común: maguey.

Hábito: hierba.

Tipos de vegetación: bosque de coníferas, matorral xerófilo.

Fuentes: Gentry, 1982 b; Espejo-Serna y López-Ferrari, 1992.

Agave polyacantha Haw. var. xalapensis (Roezl ex Jacobi) H. Gentry

Hábito: hierba.

Tipo de vegetación: bosque de coníferas.

Fuentes: Gentry, 1982 b; Espejo-Serna y López-Ferrari, 1992.

\section{ALSTROEMERIACEAE}

Bomarea gloriosa (Schltdl. \& Cham.) M. Roem.

Nombre común: canastilla, jicamilla.

Hábito: hierba.

Tipos de vegetación: bosque de Quercus, bosque mesófilo de montaña, bosque tropical perennifolio, bosque tropical caducifolio.

Fuente: Espejo y López-Ferrari, 1994a.

\section{AMARYLLIDACEAE}

Hymenocallis longibracteata Hochr.

Hábito: hierba.

Tipo de vegetación: bosque de galería.

Fuente: López-Ferrari y Espejo-Serna, 2002.

Zephyranthes miradorensis (Kraenzl.) Espejo \& López-Ferrari

Nombre común: mañanitas.

Hábito: hierba.

Tipo de vegetación: bosque tropical caducifolio.

Fuentes: Espejo-Serna y López-Ferrari, 1992; López-Ferrari y Espejo-Serna, 2002. 
Apéndice. Continuación.

\section{ANTHERICACEAE}

Echeandia albiflora (Schltdl. \& Cham.) M. Martens \& Galeotti

Hábito: hierba.

Tipos de vegetación: bosque de coníferas, bosque tropical caducifolio y bosque mesófilo de montaña.

Fuentes: Espejo-Serna y López-Ferrari, 1993; López-Ferrari y Espejo-Serna, 1995.

\section{ARACEAE}

Philodendron subincisum Schott

Hábito: hierba.

Tipo de vegetación: bosque tropical perennifolio.

Comentarios: rara.

Fuentes: Espejo-Serna y López-Ferrari, 1993; Croat, 1997.

Spathiphyllum ortgiesii Regel

Nombre común: chile de gato.

Hábito: hierba.

Tipos de vegetación: bosque de coníferas, bosque mesófilo de montaña, bosque tropical perennifolio.

Fuente: Espejo-Serna y López-Ferrari, 1993.

Spathiphyllum uxpanapense Matuda

Hábito: hierba.

Tipo de vegetación: bosque tropical perennifolio.

Fuentes: Matuda, 1976; Espejo-Serna y López-Ferrari, 1993.

Xanthosoma kerberi Engl.

Hábito: hierba.

Fuente: Espejo-Serna y López-Ferrari, 1993.

\section{ARECACEAE}

Bactris baculifera Karw.

Hábito: arbusto.

Fuente: Espejo-Serna y López-Ferrari, 1993.

\section{BROMELIACEAE}

Catopsis wawranea Mez

Hábito: hierba.

Fuente: Espejo-Serna y López-Ferrari, 1994b. 
Apéndice. Continuación.

Hechtia glabra Brandegee

Hábito: hierba.

Fuentes: Smith y Downs, 1974; García-Franco, 1987; Espejo-Serna y López-Ferrari, 1994b.

Hechtia lindmanioides L. B. Sm.

Hábito: hierba.

Tipo de vegetación: bosque tropical caducifolio.

Fuentes: Smith y Downs, 1974; García-Franco, 1987; Espejo-Serna y López-Ferrari, 1994b.

Tillandsia alvareziae Rauh

Hábito: hierba.

Fuente: Espejo-Serna y López-Ferrari, $1994 b$.

Tillandsia flabellata Baker var. viridiflora M. B. Foster

Hábito: hierba.

Fuente: Espejo-Serna y López-Ferrari, $1994 b$.

Tillandsia flavobracteata Matuda

Hábito: hierba.

Tipos de vegetación: bosque tropical perennifolio, bosque tropical caducifolio, bosque tropical subcaducifolio.

Fuentes: García-Franco, 1987; Espejo-Serna y López-Ferrari, 1994b.

Tillandsia novakii $\mathrm{H}$. Luther

Hábito: hierba.

Fuentes: Luther, 1991; Espejo-Serna y López-Ferrari, 1994b.

Tillandsia tricolor Schltdl. \& Cham.

Hábito: hierba.

Fuente: Espejo-Serna y López-Ferrari, $1994 b$.

Tillandsia viridiflora (Beer) Baker var. variegata Seaborn

Hábito: hierba.

Fuente: Espejo-Serna y López-Ferrari, 1994b.

COMMELINACEAE

Tripogandra silvatica Handlos

Hábito: hierba.

Fuentes: Handlos, 1975; Espejo-Serna y López-Ferrari, 1995. 
Apéndice. Continuación.

\section{CONVALLARIACEAE}

Maianthemum macrophyllum (M. Martens \& Galeotti) LaFrankie

Hábito: hierba.

Tipos de vegetación: bosque de Quercus, bosque mesófilo de montaña, bosque de coníferas, bosque de galería.

Fuentes: López-Ferrari y Espejo, 1993; Espejo-Serna y López-Ferrari, 1995.

\section{COSTACEAE}

Costus dirzoi García-Mendoza \& Ibarra-Manríquez

Nombres comunes: bordón, caña agria, caña de venado.

Hábito: hierba.

Tipos de vegetación: bosque tropical perennifolio, bosque de galería.

Fuentes: Vovides, 1994; Espejo-Serna y López-Ferrari, 1995.

\section{CYPERACEAE}

Carex ballsii Nelmes

Hábito: hierba.

Fuente: Espejo-Serna y López-Ferrari, 1997a.

Scleria hirta Boeck.

Hábito: hierba.

Fuente: Espejo-Serna y López-Ferrari, 1997a.

\section{DIOSCOREACEAE}

Dioscorea cruzensis R. Knuth

Hábito: bejuco.

Tipos de vegetación: bosque tropical perennifolio, bosque de galería.

Fuentes: Sosa et al., 1987; Espejo-Serna y López-Ferrari, 1996.

Dioscorea orizabensis Uline ex Knuth

Hábito: bejuco.

Tipo de vegetación: bosque mesófilo de montaña.

Fuentes: Sosa et al., 1987; Espejo-Serna y López-Ferrari, 1996.

Dioscorea spiculiflora Hemsley var. fasciculocongesta V. Sosa \& Schubert Hábito: bejuco.

Tipo de vegetación: bosque tropical subcaducifolio.

Fuentes: Sosa y Schubert, 1986; Espejo-Serna y López-Ferrari, 1996.

Dioscorea spiculoides Matuda

Hábito: hierba.

Fuentes: Matuda, 1953; Espejo-Serna y López-Ferrari, 1996. 
Apéndice. Continuación.

ERIOCAULACEAE

Paepalanthus mellii Moldenke

Hábito: hierba.

Fuente: Espejo-Serna y López-Ferrari, 1996.

IRIDACEAE

Alophia veracruzana Goldblatt \& T. M. Howard

Hábito: hierba.

Tipos de vegetación: bosque tropical perennifolio, vegetación de dunas costeras.

Fuentes: Espejo-Serna y López-Ferrari, 1996; Espejo-Serna y López-Ferrari, 1998a.

\section{MARANTACEAE}

Calathea misantlensis Lascurain

Nombre común: papatlillo, platanillo.

Hábito: hierba.

Tipos de vegetación: bosque mesófilo de montaña, bosque tropical perennifolio.

Uso: para envolver tamales.

Comentarios: solamente se ha registrado en las Sierras de Otontepec y de Chiconquiaco.

Fuente: Lascurain, 1996.

Stromanthe popolucana Castillo-Campos, Vovides \& Vázquez Torres

Hábito: hierba.

Tipo de vegetación: bosque tropical perennifolio.

Comentarios: amenazada.

Fuente: Castillo-Campos et al., 1998a.

ORCHIDACEAE

Epidendrum dressleri Hágsater

Hábito: hierba.

Tipo de vegetación: bosque tropical perennifolio.

Fuentes: Espejo-Serna y López-Ferrari, 1997 b; García-Cruz y Sánchez, 1999.

Epidendrum stallforthianum Kraenzl.

Hábito: hierba.

Tipo de vegetación: bosque de Quercus.

Fuentes: Espejo-Serna y López-Ferrari, 1997b; García-Cruz y Sánchez, 1999.

Gongora saccata Rchb. f.

Hábito: hierba.

Fuente: Espejo-Serna y López-Ferrari, 1997b. 
Apéndice. Continuación.

Liparis lindeniana (A. Rich. \& Galeotti) Hemsl.

Hábito: hierba.

Fuente: Espejo-Serna y López-Ferrari, 1998b.

Mormodes tuxtlensis Salazar

Hábito: hierba.

Tipo de vegetación: bosque tropical perennifolio.

Uso: fines comerciales.

Fuentes: Salazar, 1988; Espejo-Serna y López-Ferrari, 1998 b.

Oncidium stramineum Bateman ex Lindl.

Hábito: hierba.

Tipo de vegetación: bosque mesófilo de montaña.

Fuentes: Hawkes, 1965; Hietz y Hietz-Seifert, 1994; Espejo-Serna y López-Ferrari, 1998 b.

Ornithocephalus iridifolius Rchb. f.

Hábito: hierba.

Tipo de vegetación: bosque mesófilo de montaña.

Fuente: Espejo-Serna y López-Ferrari, 1998b.

Pleurothallis violacea A. Rich. \& Galeotti

Hábito: hierba.

Fuente: Espejo-Serna y López-Ferrari, 1998 b.

Schiedeella pubicaulis (L. O. Williams) Burns-Bal.

Hábito: hierba.

Fuentes: Balogh, 1981; Espejo-Serna y López-Ferrari, 1998 b.

Vanilla sativa Schiede

Hábito: hierba.

Fuente: Espejo-Serna y López-Ferrari, 1998b.

Vanilla sylvestris Schiede

Hábito: hierba.

Fuente: Espejo-Serna y López-Ferrari, 1998b.

POACEAE

Aristida fournieriana Hitchc.

Hábito: hierba.

Fuentes: Beetle et al., 1995; Espejo-Serna y López-Ferrari, 2000a. 
Apéndice. Continuación.

Axonopus multipes Swallen

Hábito: hierba.

Tipo de vegetación: pastizal.

Uso: forrajero.

Fuentes: Beetle y Miranda, 1983; Espejo-Serna y López-Ferrari, 2000a.

Chusquea mulleri Munro

Hábito: hierba.

Fuente: Espejo-Serna y López-Ferrari, 2000a.

Festuca bidenticulata E. B. Alexeev

Hábito: hierba.

Tipo de vegetación: bosque de coníferas.

Fuente: Espejo-Serna y López-Ferrari, 2000b.

Muhlenbergia laxa Hitchc.

Hábito: hierba.

Tipos de vegetación: bosque tropical perennifolio, bosque tropical caducifolio, bosque tropical subcaducifolio.

Fuentes: Beetle et al., 1995; Espejo-Serna y López-Ferrari, 2000 b.

Olmeca recta Soderstr.

Hábito: arbusto.

Tipo de vegetación: bosque tropical perennifolio.

Fuentes: Soderstrom, 1982; Beetle et al., 1995; Espejo-Serna y López-Ferrari, 2000b.

Panicum longum Hitchc. \& Chase

Hábito: hierba.

Fuentes: Hitchcock y Chase, 1910; Espejo-Serna y López-Ferrari, 2000b.

Schizachyrium muelleri Nash

Hábito: hierba.

Fuente: Espejo-Serna y López-Ferrari, 2000c.

\section{SMILACACEAE}

Smilax paniculata M. Martens \& Galeotti

Hábito: hierba.

Fuente: Espejo-Serna y López-Ferrari, 2000c. 
Apéndice. Continuación.

\section{DICOTYLEDONEAE}

\section{ACANTHACEAE}

Ruellia tuxtlensis T. P. Ramamoorthy \& Hornelas

Hábito: hierba.

Tipo de vegetación: bosque de galería.

Fuente: Ibarra-Manríquez y Sinaca, 1995.

\section{ARISTOLOCHIACEAE}

Aristolochia asclepiadifolia Brandeg.

Nombres comunes: guaco, patito, raíz de guaco.

Hábito: liana.

Tipos de vegetación: bosque tropical caducifolio, bosque tropical perennifolio, bosque de Quercus, bosque de galería.

Uso: medicinal (se utiliza la raíz o algunas veces el tallo en infusión de alcohol para tratar la mordedura de serpientes o para combatir la disentería).

Fuente: Ortega y Ortega, 1997.

Aristolochia impudica J. Ortega

Nombres comunes: guaco, sauco de montaña.

Hábito: arbusto.

Tipos de vegetación: bosque tropical perennifolio, bosque de coníferas, bosque de galería. Uso: medicinal (la corteza se emplea para el dolor de estómago).

Fuente: Ortega y Ortega, 1997.

\section{Aristolochia veracruzana J. Ortega}

Nombre común: guaco.

Hábito: liana.

Tipos de vegetación: bosque tropical perennifolio, bosque de Quercus, bosque de galería. Usos: medicinal (se utilizan la raíz, el tallo y las hojas para el tratamiento de la disentería y dolores estomacales); es antídoto para la mordedura de serpientes.

Fuente: Ortega y Ortega, 1997.

\section{ASTERACEAE}

Hidalgoa uspanapa Turner

Hábito: bejuco.

Tipo de vegetación: bosque de galería.

Fuente: Turner, 1988.

Loxothysanus filipes B. L. Rob.

Hábito: hierba.

Fuente: Missouri Botanical Garden's VAST, 1995. 
Apéndice. Continuación.

\section{BALSAMINACEAE}

Impatiens mexicana Rydb.

Hábito: hierba.

Tipo de vegetación: bosque mesófilo de montaña.

Comentarios: especie rara, sólo se ha colectado en las barrancas húmedas de Orizaba y Cofre de Perote.

Fuente: Barringer, 1991.

\section{BEGONIACEAE}

Begonia hydrocotylifolia Otto ex Hook.

Hábito: hierba.

Tipos de vegetación: bosque mesófilo de montaña, bosque tropical caducifolio.

Fuente: Jiménez y Schubert, 1997.

Begonia imperialis Lem.

Hábito: hierba.

Tipo de vegetación: bosque tropical perennifolio.

Fuente: Jiménez y Schubert, 1997.

Begonia lyniceorum Burt-Utley

Hábito: hierba.

Tipo de vegetación: bosque tropical subcaducifolio.

Fuente: Jiménez y Schubert, 1997.

Begonia multistaminea Burt-Utley

Nombres comunes: begonia, chucuyule y xucuyule (Veracruz).

Hábito: hierba.

Tipos de vegetación: bosque de Quercus, bosque mesófilo de montaña, bosque de coníferas. Uso: comestible (se comen los tallos).

Fuente: Jiménez y Schubert, 1997.

Begonia polygonata Liebm.

Hábito: hierba.

Tipo de vegetación: bosque tropical caducifolio.

Fuente: Jiménez y Schubert, 1997.

Begonia pudica L. B. Sm. \& Schubert

Hábito: hierba.

Tipo de vegetación: bosque mesófilo de montaña.

Fuente: Jiménez y Schubert, 1997. 
Apéndice. Continuación.

Begonia sousae Burt-Utley

Nombre común: mano de león (Veracruz).

Hábito: hierba.

Tipos de vegetación: bosque tropical perennifolio, bosque tropical subcaducifolio.

Fuente: Jiménez y Schubert, 1997.

BIGNONIACEAE

Amphitecna tuxtlensis A. Gentry

Nombre común: jicarillo.

Hábito: árbol.

Tipos de vegetación: dunas costeras, bosque mesófilo de montaña, bosque tropical perennifolio.

Usos: comestible, medicinal.

Fuente: Gentry, 1982a.

BOMBACACEAE

Quararibea yunckeri Standl. subsp. veracruzana W. S. Alverson

Nombre común: huacanelo, molinillo.

Hábito: árbol.

Tipo de vegetación: bosque mesófilo de montaña.

Uso: elaboración de mangos de herramientas.

Comentarios: muy escasa.

Fuente: Avendaño, 1998.

BORAGINACEAE

Tournefortia pedicellata D. Nash

Hábito: arbusto.

Fuente: Nash y Moreno, 1981.

BURSERACEAE

Bursera cinerea Engl.

Nombres comunes: camarón, camaroncillo, copalillo; palo mulato (Veracruz).

Hábito: árbol.

Tipo de vegetación: bosque tropical caducifolio.

Uso: medicinal (la corteza se emplea contra afecciones de las vías urinarias).

Comentarios: es similar a B. simaruba en su corteza rojiza exfoliante, varios caracteres de los folíolos, flores e inflorescencia.

Fuente: Rzedowski y Calderón, 1996. 
Apéndice. Continuación.

\section{CACTACEAE}

Coryphantha greenwoodii Bravo

Hábito: hierba.

Tipos de vegetación: bosque de coníferas, pastizal.

Fuente: Bravo-Hollis, 1970.

Mammillaria eriacantha Link \& Otto

Hábito: hierba.

Tipo de vegetación: bosque tropical caducifolio.

Uso: ornamental.

Fuentes: Bravo-Hollis, 1937; Britton y Rose, 1963.

Mammillaria sartorii J. A. Purpus

Hábito: hierba.

Tipo de vegetación: bosque tropical caducifolio.

Uso: ornamental.

Fuentes: Bravo-Hollis, 1937; Britton y Rose, 1963.

\section{CELASTRACEAE}

Euonymus platyphyllus Lundell

Hábito: árbol.

Tipo de vegetación: bosque tropical perennifolio.

Fuente: Lundell, 1984c.

\section{CONVOLVULACEAE}

Evolvulus choapanus McDonald

Hábito: hierba.

Tipo de vegetación: sabana.

Comentarios: representa la única especie de la sección Phyllostachyi Meissn. que se encuentra en Norteamérica, siendo un grupo cuyo centro de diversidad está en Brasil. Fuente: McDonald, 1993.

Ipomoea eximia House

Hábito: bejuco.

Comentarios: es una especie rara, y débilmente diferenciada de I. ignava, de la cual se distingue por poseer sépalos y corolas más pequeñas y hojas deltoide-reniformes.

Fuente: McDonald, 1994. 
Apéndice. Continuación.

\section{DICHAPETALACEAE}

Dichapetalum mexicanum Prance

Hábito: árbol.

Tipo de vegetación: bosque mesófilo de montaña.

Fuente: Durán-Espinoza, 1997.

\section{FABACEAE}

Bauhinia jucunda Brandegee

Hábito: arbusto.

Tipos de vegetación: bosque tropical caducifolio, bosque tropical subcaducifolio.

Fuente: Wunderlin, 1983.

Inga lacustris Sousa

Hábito: árbol.

Tipo de vegetación: bosque tropical perennifolio.

Fuente: Pennington, 1997.

Inga sinacae Sousa \& Ibarra-Manríquez

Nombre común: vaina peluda.

Hábito: árbol.

Tipo de vegetación: bosque tropical perennifolio.

Fuente: Pennington, 1997.

\section{HYDRANGEACEAE}

Deutzia mexicana Hemsl.

Hábito: arbusto.

Tipos de vegetación: bosque de Quercus, bosque de coníferas.

Fuente: Durán-Espinosa, 1999.

Hydrangea nebulicola Nevling \& Gómez-Pompa

Hábito: liana.

Tipos de vegetación: bosque mesófilo de montaña, bosque tropical perennifolio.

Fuente: Durán-Espinosa, 1999.

HYDROPHYLLACEAE

Hydrolea ovata var. parvifolia D. Nash

Hábito: hierba.

Tipo de vegetación: bosque tropical perennifolio.

Fuente: Nash, 1979. 
Apéndice. Continuación.

Nama linearis D. Nash

Hábito: hierba.

Tipo de vegetación: bosque de Quercus.

Fuente: Nash, 1979.

Nama orizabensis D. Nash

Hábito: hierba.

Fuente: Nash, 1979.

\section{JUGLANDACEAE}

Alfaroa mexicana D. E. Stone

Nombres comunes: cash (popoluca), cedrillo, palo de cedrillo.

Hábito: árbol.

Tipos de vegetación: bosque mesófilo de montaña, bosque tropical perennifolio.

Fuente: Narave, 1983.

\section{LYTHRACEAE}

Cuphea nitidula $\mathrm{H}$. B. \& $\mathrm{K}$.

Nombres comunes: serpentina estrella, tulipancillo.

Hábito: hierba.

Tipos de vegetación: bosque de Quercus, bosque de galería, bosque mesófilo de montaña, bosque de coníferas, matorral xerófilo, bosque tropical perennifolio.

Usos: medicinal, ornamental.

Fuente: Graham, 1991.

MALVACEAE

Hampea integerrima Schltdl.

Nombres comunes: cucharo, jonote blanco, jonote real, jonotillo, tecolixtle, majagua.

Hábito: árbol.

Tipos de vegetación: bosque de Quercus, bosque mesófilo de montaña, bosque de coníferas, matorral xerófilo, bosque tropical perennifolio, bosque tropical subcaducifolio, bosque de galería.

Fuente: Fryxell, 1992.

Robinsonella lindeniana (Turcz.) Rose \& E. G. Baker subsp. lindeniana Nombre común: chaqueta de novia.

Hábito: arbusto.

Tipos de vegetación: bosque de Quercus, bosque mesófilo de montaña, bosque tropical perennifolio.

Uso: ornamental.

Fuente: Fryxell, 1992. 
Apéndice. Continuación.

\section{MENISPERMACEAE}

Hyperbaena jalcomulcensis E. Pérez \& Cast.-Campos

Hábito: árbol.

Tipos de vegetación: bosque tropical caducifolio, bosque tropical perennifolio, bosque tropical subcaducifolio.

Fuentes: Pérez y Castillo-Campos, 1988; Pérez, 1995.

\section{MORACEAE}

Dorstenia uxpanapana C. C. Berg \& T. Wendt

Hábito: hierba.

Tipo de vegetación: bosque tropical perennifolio.

Fuente: Missouri Botanical Garden's VAST, 1995.

\section{MYRSINACEAE}

Parathesis pajapanensis Lundell

Hábito: arbusto.

Fuente: Missouri Botanical Garden's VAST, 1995.

Parathesis tuxtlensis Lundell

Hábito: arbusto.

Fuente: Lundell, 1984 a.

\section{MYRTACEAE}

Calyptranthes karwinskyana O. Berg

Hábito: árbol.

Tipos de vegetación: bosque de Quercus, bosque mesófilo de montaña.

Fuente: Sánchez-Vindas, 1990.

\section{Calyptranthes schiedeana O. Berg}

Nombres comunes: petcoy (popoluca), mi-tsinin-qui-hui (totonaca) y guayabillo.

Hábito: árbol.

Tipos de vegetación: bosque de Quercus, bosque mesófilo de montaña, bosque tropical perennifolio, bosque tropical caducifolio, bosque tropical subcaducifolio, bosque de galería. Fuente: Sánchez-Vindas, 1990.

Calyptranthes schlechtendaliana O. Berg

Nombre común: guayabillo.

Hábito: arbusto.

Tipos de vegetación: bosque de Quercus, bosque de galería, bosque tropical perennifolio, bosque tropical caducifolio, bosque tropical subcaducifolio.

Fuente: Sánchez-Vindas, 1990. 
Apéndice. Continuación.

Eugenia colipensis O. Berg

Nombres comunes: escobillo, escobilla, viscarona.

Hábito: árbol.

Tipos de vegetación: bosque mesófilo de montaña, bosque tropical perennifolio.

Fuente: Sánchez-Vindas, 1990.

Eugenia inirebensis P. E. Sánchez

Hábito: árbol.

Tipo de vegetación: bosque tropical perennifolio.

Fuente: Sánchez-Vindas, 1990.

Eugenia ledophylla (Standley) McVaugh

Hábito: arbusto.

Tipo de vegetación: bosque tropical caducifolio.

Fuente: Sánchez-Vindas, 1990.

Eugenia mozomboensis P. E. Sánchez

Hábito: arbusto.

Tipo de vegetación: bosque tropical caducifolio.

Fuente: Sánchez-Vindas, 1990.

Eugenia sotoesparzae P. E. Sánchez

Hábito: árbol.

Tipos de vegetación: dunas costeras, bosque tropical perennifolio.

Comentarios: se caracteriza principalmente por presentarse en hábitats costeros.

Fuente: Sánchez-Vindas, 1990.

Eugenia trunciflora (Cham. \& Schldl.) O. Berg

Nombres comunes: cojón de gato, manzanita cimarrona, manzanito cimarrón, shanatkini (totonaca, Veracruz).

Hábito: árbol.

Tipos de vegetación: bosque mesófilo de montaña, bosque tropical perennifolio, bosque de galería.

Fuente: Sánchez-Vindas, 1990.

Eugenia uxpanapensis P. E. Sánchez \& L. M. Ortega

Nombres comunes: escobilla, escobilla real.

Hábito: árbol.

Tipo de vegetación: bosque tropical perennifolio.

Fuente: Sánchez-Vindas, 1990. 
Apéndice. Continuación.

RHAMNACEAE

Colubrina johnstonii Wendt

Hábito: arbusto.

Tipo de vegetación: bosque tropical perennifolio.

Fuente: Fernández, 1986.

Rhamnus capraeifolia var. matudae L. A. Johnston \& M. C. Johnston

Hábito: arbusto.

Tipos de vegetación: bosque de Quercus, bosque de coníferas.

Fuente: Fernández, 1986.

RUBIACEAE

Antirhea aromatica Cast.-Campos \& Lorence

Nombre común: chicahuastle.

Hábito: árbol.

Tipo de vegetación: bosque tropical perennifolio.

Uso: construcción y medicinal.

Fuente: Castillo-Campos y Lorence, 1985.

Rondeletia tuxtlensis Lorence \& Cast.-Campos

Hábito: árbol.

Tipo de vegetación: bosque mesófilo de montaña.

Fuente: Lorence y Castillo-Campos, 1988.

SOLANACEAE

Cestrum endlicheri Miers

Nombre común: hierba del coyote, hierba del espanto.

Hábito: arbusto.

Tipos de vegetación: bosque mesófilo de montaña, bosque de Quercus.

Uso: medicinal.

Comentarios: es considera como la más elegante y hermosa de las especies de la sección Habrothamnus.

Fuente: Nee, 1986.

Cestrum miradorense Francey

Hábito: arbusto.

Tipo de vegetación: bosque mesófilo de montaña.

Fuente: Nee, 1986. 
Apéndice. Continuación.

Physalis greenmanii Waterf.

Hábito: hierba.

Tipo de vegetación: bosque mesófilo de montaña.

Fuente: Nee, 1986.

Solanum palmillae Standl.

Hábito: arbusto.

Tipos de vegetación: bosque tropical caducifolio, bosque de galería.

Fuente: Nee, 1993.

\section{THEOPHRASTACEAE}

Jacquinia morenoana Cast.-Campos \& Medina Abreo

Nombre común: arbolito de navidad.

Hábito: arbusto.

Tipos de vegetación: bosque tropical perennifolio, vegetación de dunas costeras.

Uso: ornamental.

Fuente: Castillo-Campos y Medina, 1998b.

THYMELAEACEAE

Daphnopsis brevifolia Nevling

Hábito: árbol.

Tipo de vegetación: bosque tropical caducifolio.

Fuente: Nevling y Barringer, 1988.

Daphnopsis megacarpa Nevling \& Barringer

Hábito: árbol.

Tipos de vegetación: bosque mesófilo de montaña, bosque tropical perennifolio.

Fuente: Nevling y Barringer, 1988.

\section{VERBENACEAE}

Citharexylum bourgeauianum Greenm.

Hábito: arbusto.

Tipo de vegetación: bosque mesófilo de montaña.

Fuente: Nash y Nee, 1984.

Citharexylum fulgidum Moldenke

Hábito: arbusto.

Tipos de vegetación: bosque de Quercus, bosque de coníferas.

Fuente: Nash y Nee, 1984. 
Apéndice. Continuación.

Citharexylum kerberi Greenm.

Nombre común: aceitunillo.

Hábito: arbusto.

Tipo de vegetación: bosque tropical caducifolio.

Fuente: Nash y Nee, 1984.

VIOLACEAE

Orthion veracruzense Lundell

Hábito: árbol.

Tipo de vegetación: bosque tropical perennifolio.

Fuente: Lundell, 1984b.

Rinorea uxpanapana $\mathrm{T}$. Wendt

Hábito: árbol.

Tipo de vegetación: bosque tropical perennifolio.

Fuente: Wendt, 1983. 